\title{
DRAFT: A MATLAB-BASED APPLICATION DEVELOPMENT USING A 3D PMD CAMERA FOR A MOBILE ROBOT
}

\author{
Mohammad Chami \\ Mobile Robotics and Mechatronics Lab \\ University of Applied Sciences Ravensburg- \\ Weingarten \\ Weingarten,D-88241,Germany \\ Email: chami@hs-weingarten.de
}

\author{
Holger Voos \\ Automatic Control Laboratory \\ University of Luxembourg \\ Luxembourg, L-1359 \\ Email: holger.voos@uni.lu
}

\begin{abstract}
Three-dimensional sensing is a vital field in mobile robotic applications. This work proposes an application of a Time-ofFlight 3D Photonic Mixer Device (PMD) camera for the navigation of an Omni-directional mobile robot. The 3D PMD camera enables real time distance detection as well as the capturing of grayscale images. In our framework, the application of the 3D PMD camera is aimed at solving the problem of environmental perception in mobile robotics. In this paper, we present the development of a MATLAB-based kit for the control of an Omni-directional mobile robot supported by a data acquisition board. The communication interface of the camera, used to close the system's control loop, has been also developed. We further present results of different experiments including online obstacle detection and avoidance. In addition, an adaptive pose determination for the robot is proposed.
\end{abstract}

\section{INTRODUCTION}

The field of mobile robotics has been under an extreme interest of research in the past few years. They have been engaged in different meaningful interactive applications especially in industry, military as well as in educational and research environments. More specifically, wheeled mobile robots have been more employed in dynamic environment applications [1, 2]. In order to achieve an effective mobile robot performance, an intensive research and development is essential for the guidance, navigation and control systems. Liu emphasized in [1], the importance of a stable and accurate navigation system as a bridge between the guidance and control system, by providing the mobile robot with accurate position, velocity and/or orientation information. Since decades, engineers and researchers have developed a wide range of position sensors to extract the knowledge of a mobile robot environment. 3D sensors are getting more attention recently due to the valuable $3 \mathrm{D}$ data they can provide. A detailed discussion about existing sensors and techniques for mobile robot positioning is presented in [3].

3D sensor technologies have been categorized in $[4,5]$ into three categories: 3D laser scanners, stereo vision systems and Time-of-Flight (ToF) cameras. So far, 3D laser scanners and stereo camera systems are mostly used due to their high measurement range and precision [6]. In contrast, ToF cameras provide enormous advantage due to their high frame rate. This high frame rate has to be balanced with the measurement precisions [6]. Stereo vision systems involve high amount of computational power [7] and they need a great deal of performance for finding the correspondence point from both left and right images in order to be able to calculate the depth information [8]. Since one decade, a new generation of ToF cameras, the PMD, has been invented. PMD cameras consist of PMD sensor chips realized in CMOS technology [9]. They are called smart pixels, since within each pixel the complete mixing process of the electric and optical signal takes place $[9$, 10]. More details about the PMD operational principle can be found in [10-12].

Beyond the hardware considerations, deep software knowledge is crucial for realizing robust mobile robotic applications. MATLAB [13] is suitable for such applications since it offers a mathematical-based intuitive programming language which is supported by a wide range of toolboxes. It has been used for educational and industrial purposes and has proven effective simulation and analysis approaches.

In our framework, we choose a 3D PMD camera, the "efector PMD $3 D$ " a prototype (developed by ifm electronic 
gmbh [12]) and apply it, as the only sensor, for the control of an Omni-directional mobile robot with MATLAB running on a PC-based real-time system. After the hardware and software development of the framework kit, several obstacle avoidance experiments have been performed to verify the employment of such a 3D PMD camera in the mobile robotics field. In addition, an adaptive object recognition followed by pose determination of the robot is proposed. The limitations of this camera noticed during the work are presented. The rest of the paper is organized as follows: Section 2, offers a related work review. Section 3, describes the background work of the framework and the hardware architecture. Further on, in Section 4 we describe in details the software development part of our framework. Experimental results attained are represented in section 5. Section 6, concludes and reflects upon future work.

\section{RELATED WORK}

Three-dimensional vision applications have been spreading rapidly since the last period. Not only in industrial or automotive applications but also in several home entertainment products. Several products of 3D PMD cameras are being developed by adapting modular components on customer requirements [10]. These cameras are mainly requested by automotive and robotic industries. Ringbeck \& Hagebeuker describe in [10] the operational principle of a PMD sensor and the components of PMD camera systems. They gave flexible modular camera design examples for customer specific solutions dealing with industrial products, robotics activities and automotive applications. Fardi et al. [14] presented an application of a PMD camera in the automotive industry for obstacle detection and pedestrian recognition.

In [7] a 3D-ToF PMD camera is proposed as a robot vision system and an application is described for object segmentation algorithms compared to a stereo vision system. Hussmann et al. present in [15] an integration of a ToF sensor into an autonomous mobile robot system. Experiments and results show that the ToF sensor is more suited for robot navigation than the existing laser range system [15].

\section{BACKGROUND}

The work presented in this paper represents a running project at the University of Applied Sciences RavensburgWeingarten, dealing with different applications of mobile robotics. In previous work, a three wheeled Omni-directional mobile robot has been designed and developed as a test platform for different research applications. This mobile robot design has been used as a basis in several projects such as the Robocup competition. The robot was used in our framework as a usability verification platform for a 3D PMD camera in the mobile robotics field. Fig. 1 describes the overall scheme of our framework architecture which can be divided into three parts: the mobile robot, the 3D PMD camera and a computer used for monitoring and controlling.

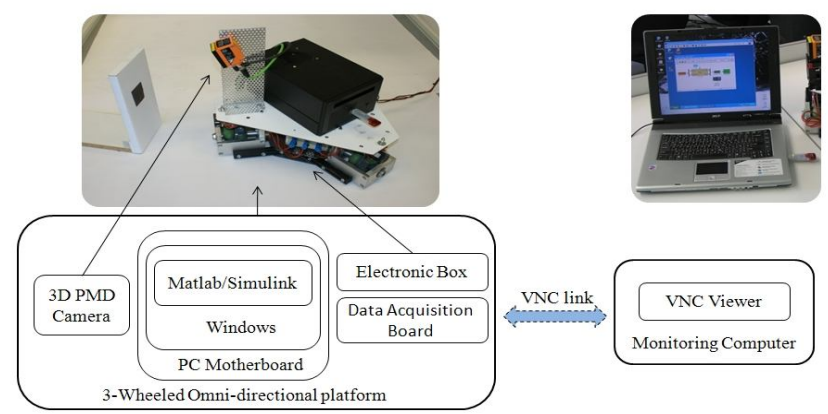

FIGURE 1. FRAMEWORK ARCHITECTURE

The Omni-directional mobile robot platform is made up of the chassis and three Swedish wheels placed $120^{\circ}$ symmetrically apart. It is supported by a motherboard with its peripherals and Windows XP as an operating system. A MATLAB-based control system kit has been developed to control the motion of the robot for different applications. A multifunctional input/output Data Acquisition Board (DAQ), MF624 developed by the company Humusoft [16], is connected to the PCI bus and used to interface MATLAB and the electronic box, which in turn contains the motors controller board and the power supply. The MF624 is supported with a MATLAB real-time toolbox offering a wide range of applications.

The 3D PMD camera, described in details in section 3.2, is mounted on the robot with an inclination angle of 45 degrees in order to capture the front area of the mobile robot for both detecting obstacles and recognizing an adaptive object. The communication between the camera and the PC is established by Ethernet and done through the LAN port of the PC. A Simulink model has been developed for the camera and integrated with the main MATLAB control system kit model.

In order to control the robot wirelessly, another computer is used to monitor the robot's PC. A Virtual Network Computing (VNC) protocol is used and as a result the remote PC's desktop is displayed on the screen of the laptop offering a full control of the robot's PC remotely. During the first stages of the software development, the work was realized in a twofold: (1) programming and controlling of the mobile robot and (2) handling the communication with the camera and applying image processing and analysis techniques. Later on, the two parts were integrated in one system model and several experiments were performed to verify the application. In the following a brief background on both the mobile robot and the 3D PMD camera is presented.

\subsection{Mobile Robot Kinematics}

One of the main advantages of Omni-directional mobile robots is that they can move at any time in any direction, this is performed by a simultaneous or independent combination of translation and rotational motion. 


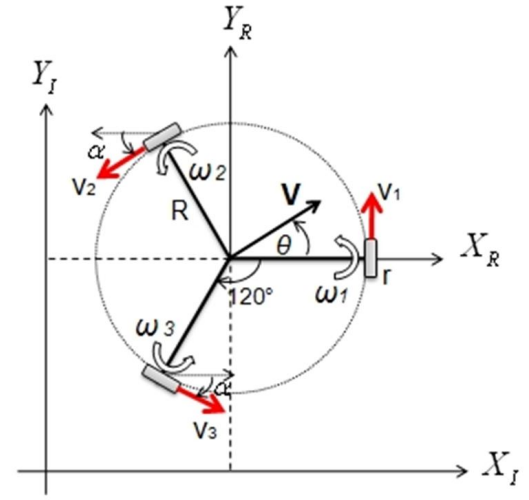

FIGURE 2. OMNI-ROBOT KINEMATIC MODEL

Figure 2 shows the kinematic diagram model of the used Omni-directional robot with two coordinate frames. The first is the global frame and denoted by $\left\{\mathrm{X}_{\mathrm{I}}, \mathrm{Y}_{\mathrm{I}}\right\}$, while the second is corresponding to the local coordinate frame and denoted by $\left\{X_{R}, Y_{R}\right\}$. The local frame is fixed on the moving robot with the origin located at its center of gravity. The $\mathrm{X}$-coordinate of movement, $X_{R}$, is fixed in the same direction of the first wheel axle. The global frame is considered to be fixed with an arbitrary inertial basis axes $X_{I}$ and $Y_{I}$ at the ground of the robot's workspace.

The inverse kinematics model of the robot can be easily determined and is expressed in Eqn. (1):

$$
\left[\begin{array}{l}
w_{1} \\
w_{2} \\
w_{3}
\end{array}\right]=\frac{1}{r} M^{-1}\left[\begin{array}{l}
V_{x} \\
V_{y} \\
W
\end{array}\right], \text { where } M=\left[\begin{array}{ccc}
0 & -\frac{\sqrt{3}}{2} & \frac{\sqrt{3}}{2} \\
1 & -\frac{1}{2} & -\frac{1}{2} \\
R & R & R
\end{array}\right]
$$

where $w_{1}, w_{2}$ and $w_{3}$ represent the angular velocities of each of the three wheels respectively. $V_{x}, V_{y}$ and $W$ represent the $\mathrm{x}, \mathrm{y}$ velocity components and the rotational speed of the robot respectively. $M$ is the transformation matrix deduced by the comparison between $w_{i}$ and the projection of robot's speed $V$ and rotation $W . R$ and $r$ represent the radius of the robot and the wheels respectively. Eqn. (1) is written in a MATLAB Mfile and used in the system's model to transform the requested velocity and rotation motion of the robot to the angular velocity-values of each of the wheels.

\subsection{D PMD Camera}

The efector $P M D 3 D$ shown in Fig. 1 is a $3 \mathrm{D}$ image sensor that uses Photonic Mixing Device (PMD) Principle based on time-of-flight measurement for measuring the distance and the grayscale values for each pixel of its scene [12]. The camera has a built in PMD sensor with a resolution of $64 \times 50$ pixels and is able to capture a complete three dimensional image in real time with a variable frame rate up to $20 \mathrm{~Hz}$. The camera's field of view is $30^{\circ}$ horizontal and $40^{\circ}$ vertical and it can detect distances with an unambiguous unique range of $7.5 \mathrm{~m}$ of $20 \mathrm{MHz}$ modulation frequency. More information about the unambiguous range and its relation with the modulation frequency for 3D cameras is described in [8].

The time-of-flight principle of PMD cameras is described in $[8,9]$. In its simplest form, a modulated infrared pulse light is radiated from the camera. This light is propagated through its scene until it is reflected by an object. The reflected light is then collected back by the lens and detected with the camera's PMD pixels. The phase shift between the modulated and the light signal detected is measured by every pixel thus the distance to the object is determined. Background illumination is still one of the crucial aspects of ToF cameras. The PMD camera is supported with a suppression of background illumination which tends in an increase in its dynamic range and makes it suitable for outdoor applications [17]. The measured distance is proportional to the collected light, thus the accuracy of the distance measurements improves with higher collected light levels [12].

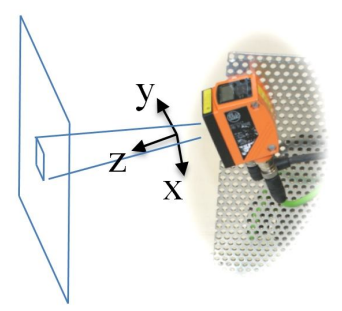

\section{FIGURE 3. 3D PMD CAMERA}

The z-axis of the camera's frame of reference is considered to be perpendicular to the camera's front surface as shown in Fig. 3. The pixels size and the field of view dimensions are directly proportional to the distance between the camera and the object. This enables the measurement of the size (width and high) and the position of objects. These measurements are demonstrated with an object recognition experiment described in section 5.1. The depth measurement with PMD cameras depends on the amplitude change in the received modulated light signal, for example, measuring a plane object with different surface colors results in different range values. This amplitude dependency can be avoided using a phase-shift algorithm [7].

\section{THE SYSTEM MODEL}

Figure 4 shows the Simulink system model created to control the mobile robot. The whole system model is an integration of three subsystems: (1) the 3D PMD camera model, used as a feedback input for the (2) main control model which is realized with a Stateflow chart with an output to (3) 
the Omni-directional robot model. In the following the control system model, the camera model and the Omni-drive robot model are described more in details.

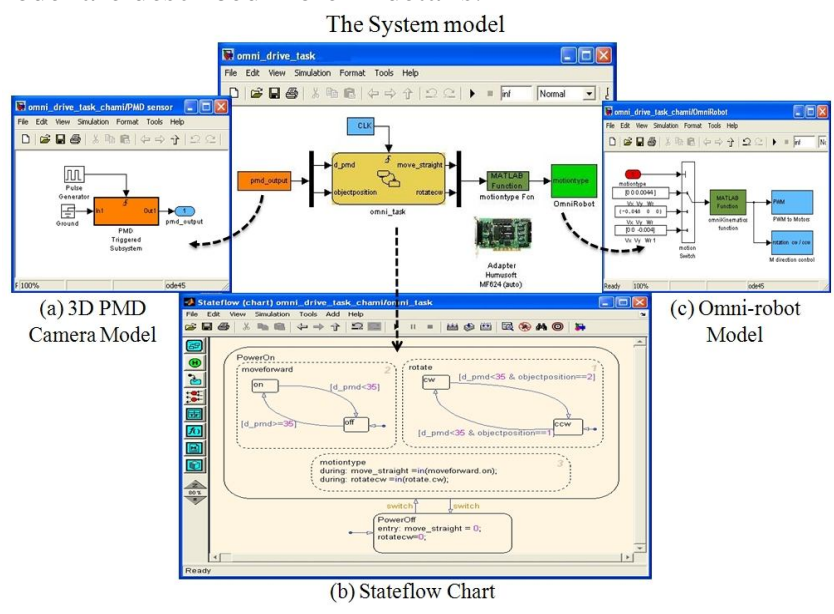

FIGURE 4. THE SIMULINK SYSTEM MODEL

\subsection{The Stateflow Chart:}

One of the main goals of the system model was to test the reliability of the real-time qualifications for such a PC-based control system. Stateflow is a powerful graphical design and simulation tool developed by Mathworks for control and supervisory logic used in conjunction with Simulink [13]. A Stateflow chart, as an example of a finite state machine, was chosen during the development period due to the time execution aspects for some logical states. These logical states describe tasks the robot had to perform. It uses the output from the camera model and sends a respective state value for the robot model specifying a direction of motion. The Stateflow chart shown in Fig. 4-b has two inputs:

- DistancePmd: the minimum distance value that the camera is detecting, this value represents the nearest distance point of the robot scene.

- ObjectPosition: a logical value indicating the position of the obstacle if it is on the right or the left side of the robot.

and two outputs:

- MoveStraight: a logical value used to command the Omni-robot to stop or move in a forward direction.

- Rotate Cw: a logical value used to command the robot to rotate in both rotational directions.

For defining the states within the stateflow chart, a finite state machine is used. In our case here, a simple task was developed to make the robot move in a forward direction and simultaneously detect obstacles and avoids them. When an obstacle is detected, its position is analyzed and an opposite rotation is carried out to avoid the obstacle till the robot can move again in forward direction to reach its target. A priority was given to the robot to rotate clockwise when it detects a plane obstacle with the whole camera field of view. The Stateflow chart is developed with two main states configured with a XOR state relation in order to work once in a time: the first state "PowerOff" initializes the system with no outputs and waits for the camera initialization. After a valid value from the camera is received a transition to the second state "PowerOn" is executed. Within the PowerOn state, three states are configured to run with an AND state relation i.e.: they run simultaneously with a neglected delay in between. These states detect the value received from the camera (DistancePmd), if it is greater than $350 \mathrm{~mm}$, the MoveStraight output is assigned to logic 1 and the robot will be moving forward. When the value is less than $350 \mathrm{~mm}$, the MoveStraight is assigned to logic 0 and the Rotate $C w$ state is assigned to 1 or 0 commanding the robot to rotate in a reversed direction to the obstacle until the way is free again to continue to the target position.

\subsection{The 3D PMD Camera Model}

The 3D PMD camera model subsystem plays the feedback role for the overall control loop by capturing the scene of the robot and providing the distance measurement. The camera is supported with a Dynamic-link library file for the interfacing with MATLAB. A triggered subsystem block was created for the camera with a $20 \mathrm{~Hz}$ frame rate and an output specifying the actual image queried in a matrix format (Fig. 4-a). In addition, a MATLAB M-file was written to perform the communication protocols and the image processing techniques.

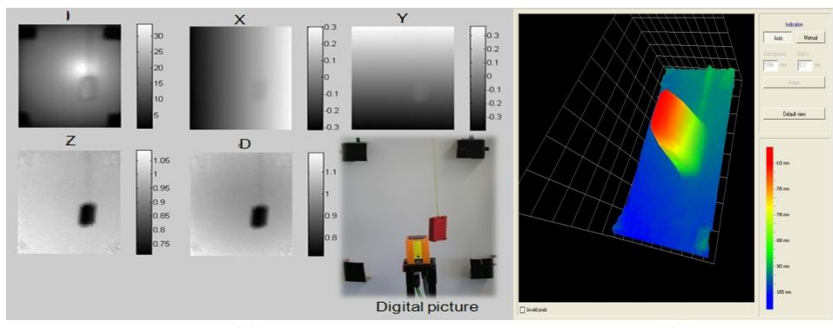

(b)

FIGURE 5. PMD CAMERA OUTPUT

a) MATLAB b) IFM Electronics Software

As mentioned in section 3.2, the 3D PMD camera determines the distance and the grayscale value of each pixel in the scene. A test platform was used to calibrate the camera for our framework requirements. The camera was placed against a wall within the range of $0.5 \mathrm{~m}$ to $6 \mathrm{~m}$. Fig. 5 shows the output of the camera placed at a $1 \mathrm{~m}$ distance from the wall with a moving object in its field of view. The driver software supported by the camera (Fig. 5-b) offers a flexible application for understanding how the camera works with its functionalities and limitations with different environments. Fig. 5-b shows the output of the camera software with the color-bar indicating the distance to the sensor with its 
corresponding color. The red color indicates the actual position of the object $(0.6 \mathrm{~m})$ with respect to the camera and the blue background represents the wall $(1 \mathrm{~m})$. Fig. 5-a indicates five different images ( $I, X, Y, Z$ and $D$ ), each with $64 \times 50$ pixels resolution attained from the camera through MATLAB and a digital picture of the test platform. The images requested are as follows:

1. I-image: is a gray scale intensity image that visualizes the reflectivity from the scene. It is similar to a black and white camera photo. Light tones for high intensity pixels of white surfaces with high reflectivity and dark tones for low intensity pixels of black surfaces with low reflectivity.

2. X-image: indicates the Cartesian x-pixel coordinates with a range from -25 to +25 pixel unit.

3. Y-image: indicates the Cartesian y-pixel coordinates with a range of -32 to +32 pixel unit.

4. Z-image: indicates the Cartesian z-pixel coordinates where the actual distance of the object with respect to the sensor surface is determined in meters.

5. D-image: indicates the radial distance value captured by the camera within an internal calculation. This matrix was used for the obstacle detection and avoidance experiment described more in details in section 5.3.

\subsection{The Omni-directional Robot}

The DAQ implemented in the Omni-directional mobile robot is supported with a real time Simulink toolbox package that connects the software model with the real world actuators and sensors. The real time toolbox contains a library of blocks used to command the three motors with its respective PWM signal to move the robot. Referring to the robot kinematics model described in section 3.1, a subsystem Simulink block (Fig. 4-c) for the robot was created with the input from the Stateflow chart describing the motion types: moving forward, rotating in clockwise or in counterclockwise direction and stop if it reaches its target position. In addition, the robot model contains a MATLAB function block that executes the M-file of the inverse kinematics equations with an output of the desired angular velocity of each wheel in the form of a PWM signal.

\section{Experiments}

The 3D PMD camera used in our work is not considered to be only a 3D camera but also a sophisticated sensor due to the gray scale image, the 3D data it provides, and its internal embedded system design. After being able to communicate and configure the camera via MATLAB, several experiments were handled on the intensity image of distinct objects with different colors and on different distances. Since the camera measurement is based on the reflected modulated light, black objects with low reflectivity can be detected up to $2 \mathrm{~m}$. Moreover, the measurements are influenced with a strong external light.

\subsection{Object Recognition}

After interfacing the camera and integrating it with the mobile robot, a useful experiment was demonstrated to recognize a defined object and deduce its position. Our defined object shown in Fig. 1 is a black spot of $100 \mathrm{~mm} \times 100 \mathrm{~mm}$ in the middle of the upper half part of an A4 white paper.

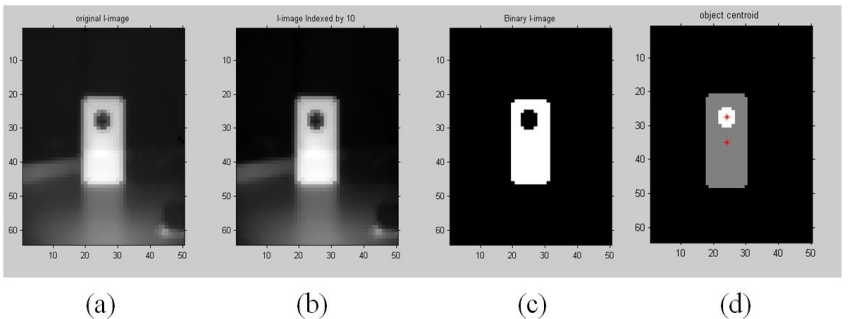

FIGURE 6. IMAGE PROCESSING AND ANALYSIS

a) Original image b) Indexed image c) Binary image d) Object detection \& centroid calculation

The object recognition process starts with suppressing the noise of the original gray scale image from the camera (Fig. 6a). The noise could be generated from a strong light source or very weak and intense gray values. Then, the image is scaled with an index of size 2 and so the gray values are doubled, this tends to enhance the recognition our object (Fig. 6-b). The index 2 and other recognized noise values were known after analyzing the intensity image in its matrix format. After having the indexed image, an extended-maxima transform function [12] is used with a value of 100 (the limit 100 was deduced from the early intensity measurement experiments). Later on, the exterior boundaries as well as the holes inside the object are traced and a binary image is obtained. A final object recognition task is performed by testing the dimensions of the white area and the black spot and comparing it with the real object. As a consequence the object was detected in real time with a maximum range of $2 \mathrm{~m}$ and with different angles in the camera's field of view.

\subsection{D Pose Determination}

After the object has been recognized (Fig. 6-c) the centroid of the black spot and the pixel coordinates of this centroid are determined. At this stage we use the I-image to detect the object and calculate the pixel coordinates of the black spot in the I-image. The next step was to access the values of the centroid-pixel from the other three images: $X$ image, $Y$-image and the Z-image. The Z-image values are determined with meters. However, the $X$-image and $Y$-image values are in pixel units, so a conversion from these units to the actual measurement in meters was done with the respective the Z-image value. 


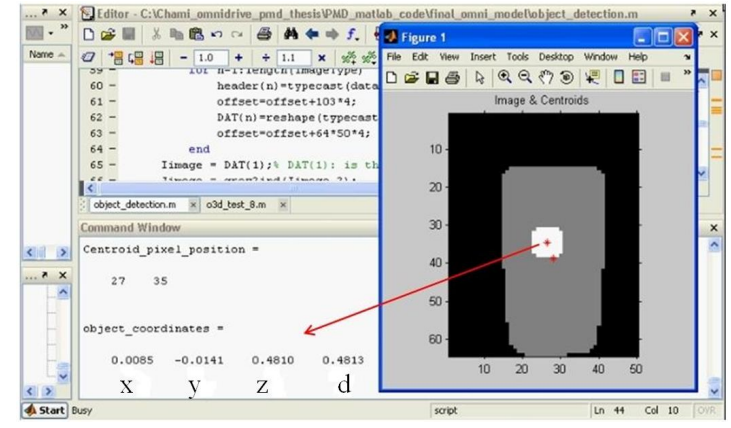

FIGURE 7. REAL-TIME POSE DETERMINATION

As a result, the $3 \mathrm{D}$ pose of the mobile robot with respect to the object was determined by an inverse transformation of the data obtained of the coordinates $x, y$ and $z$. Fig. 7 shows a sample of a real-time execution of this 3D pose determination with MATLAB on the monitoring computer. First, the command window indicates the pixel coordinates of the centroid $(27,35)$ with respect to the image frame of reference. This means that our object is recognized. Second, the 3D coordinates of the object $\mathrm{x}, \mathrm{y}$, and $\mathrm{z}$ are indicated as well as the radial distance of the black spot provided from the $D$-image.

\subsection{Obstacle Avoidance}

In order to prove the feedback reliability of the sensor for our mobile robot platform and to close the loop of the control system, an autonomous task was performed to command the robot to move with a forward direction in a dynamic environment and each time toward a different target position. While the robot is moving it should detect obstacles and avoid them. As described in the Stateflow chart states in section 4.1 and using the radial distance value from the camera $(D$ image), the robot showed a reliable performance in avoiding obstacles. Moreover, the position of the robot was determined every time the defined object was detected.

\section{CONCLUSION}

In this paper, the employment of a 3D PMD camera for the navigation of an Omni-directional mobile robot was established. Programming development with MATLAB/Simulink for the camera and the Omni-directional mobile robot was implemented and integrated in one model. During the work it was realized that working with such a sensor is not that complex and doesn't require that much computational effort compared to stereo vision cameras. The distance calculation is simply captured and provided. For future work, a 2D camera could be combined to the framework to provide high resolution images for better object recognition. Solutions should be investigated regarding the camera limitations and noise issues for outdoor applications. This camera is also aimed at a 3D map construction.

\section{REFERENCES}

[1] Liu, Y., 2010. Robot Vision. In-Tech, Olajnica 19/2, 32000 Vukovar, Croatia, Chap. 8, pp. $129-142$.

[2] Pin, F. G. and Killough, S. M. 1994. "A New Family of Omnidirectional and Holonomic Wheeled Platforms for Mobile Robots" IEEE Transactions on Robotics and Automation 10(4), 480-489.

[3] Borenstein, J., Everett, H.R., Feng, L., and Wehe D. "Mobile Robot Positioning - Sensors and Techniques". Invited paper for the Journal of Robotic Systems, Special Issue on Mobile Robots. Vol. 14 No. 4, pp. 231 - 249.

[4] Hussmann, S. and Edeler, T. 2010. Robot Vision. In-Tech, Olajnica 19/2, 32000 Vukovar, Croatia, Chap. 16, pp. 293 $-306$.

[5] Poppinga, J., Birk, A. and Pathak, K. 2009. "A Characterization of 3D Sensors for Response Robots". In Proceedings of RoboCup, pp. 264-275.

[6] May, S., Droeschel, D., Holz, D., Wiesen, C., and Fuchs, S. 2008 "3D Pose Estimation and Mapping with Time-ofFlight Cameras" In IEEE/RSJ International Conference on Intelligent Robots and Systems (IROS), Workshop on 3D Mapping, Nice, France.

[7] Hussmann, S., and Liepert, T., 2007. "Robot Vision System based on a 3D-TOF Camera". In Proceedings of the Instrumentation and Measurement Technology Conference IMTC. IEEE.

[8] May, S., Pervoelz, K. and Surmann, H. 2007. Vision Systems: Applications. In-Tech, Vienna, Austria, Chap. 11, pp. 181-202.

[9] Möller, T., Kraft, H., Frey, J., Albrecht, M. and Lange, R., 2005. Robust 3D Measurement with PMD Sensors.In: Proceedings of the 1st Range Imaging Research Day at ETH Zurich, Zurich, Switzerland.

[10] Ringbeck, T., and Hagebeuker, B., 2007. "A 3D Time of flight camera for object detection". Optical 3-D Measurement Techniques, ETH Zürich.

[11] PMD Technologies GmbH, at http://www.pmdtec.com/

[12] IFM Electronics GmbH, at http://www.ifm.com/

[13] MATLAB®, at www.mathworks.com/

[14] Fardi, B., Dousa, J., Wanielik, G., Elias, B. and Barke, A., 2006. Obstacle Detection and Pedestrian Recognition Using A 3D PMD Camera. In Proceedings of the Intelligent Vehicles Symposium, pp.225 - 230.

[15] Hussmann, S., Schauer, D. and MacDonald, B., 2009. "Integration of a 3D-TOF camera into an autonomous mobile robot system", IEEE Instrumentation and Measurement Society, I2MTC, Singapore, pp.547-552.

[16] Humosoft s.r.o., at http://www.humusoft.cz/

[17] Frey, V., 2010. PMD Cameras for Automotive \& Outdoor Applications. PMD[vision] ${ }^{\circledR}$ Day Vol. 3. Munich, Germany, at http://spectronet.de/ 